\title{
Observers for linear distributed-parameter systems: A survey*
}

\author{
Z. Hidayat, R. Babuška, B. De Schutter, and A. Núñez
}

If you want to cite this report, please use the following reference instead:

Z. Hidayat, R. Babuška, B. De Schutter, and A. Núñez, "Observers for linear distributed-parameter systems: A survey," Proceedings of the 2011 IEEE International Symposium on Robotic and Sensors Environments (ROSE 2011), Montreal, Canada, pp. 166-171, Sept. 2011.

Delft Center for Systems and Control

Delft University of Technology

Mekelweg 2, 2628 CD Delft

The Netherlands

phone: +31-15-278.24.73 (secretary)

URL: https: //www.dcsc.tudelft.nl 


\title{
Observers for Linear Distributed-Parameter Systems: A Survey
}

\author{
Z. Hidayat, R. Babuška, B. De Schutter, A. Núñez
}

\begin{abstract}
This paper reviews different observer design methods for linear dynamic distributed-parameter systems. In such systems, the states, inputs, and outputs depend on some spatial variable. This dependence, along with additional aspects such as the boundary conditions, increase the complexity of the state estimation problem and of the design methods. The paper in particular surveys observers for first-order and second-order linear distributed-parameter systems based on their infinitedimensional and finite-dimensional descriptions.
\end{abstract}

\section{INTRODUCTION}

In many real-world problems, the states, inputs, and outputs of a mathematical model of a system depend on a spatial variable, which is usually a position in a one-dimensional or a multi-dimensional space. This kind of systems are called distributed-parameter systems (DPSs), in contrast to lumpedparameter systems, whose variables do not depend on spatial parameters. Examples of DPSs can be found in process control, e.g., robotics, bio-reactors, glass feeders, biomedical engineering, flexible structures, and vibrations. An overview of DPS applications can be found in [1].

For the operation of a control system, the knowledge of states of the system is important. In most cases it is not possible to have full information of the system's states due to the fact that not all of the variables can be measured. Installing all the necessary sensors may not be physically possible or the costs may become prohibitive. In such a case, the states can be estimated using state estimators (observers). One of the basic state estimators for linear, lumped-parameter systems is the Luenberger observer [2]. This type of observer has attracted much attention and various design methods have been proposed for both the linear and the nonlinear cases For surveys on observer design methods for lumped-parameter systems, see, e.g., [3] for nonlinear observers and [4] for sliding-mode observers.

The control and state estimator design for DPSs is more complex than in the lumped-parameter case [5]-[8]. The presence of spatial variables imposes limitations to the design, e.g., observation and/or actuation may occur at the boundaries only. Research on observer design for DPSs has not been so extensive as in the case of lumped-parameter systems. Furthermore, papers on distributed-parameter observers are scattered in the literature and, to the authors' best knowledge, there are no surveys on this topic. This paper aims at filling this gap. We survey the techniques that are currently available for

The authors are with Delft Center for Systems and Control, Delft University of Technology, Mekelweg 2, 2628 CD Delft, The Netherlands Email: \{z.hidayat; r.babuska; b.deschutter; a.a.nunezvicencio\}@ tudelft.nl the design of observers for first-order and second-order linear DPSs. In addition, we identify challenges for future research in this field.

This paper is organized as follows. After an introduction in Section I, a brief review of the observer design problem for lumped-parameter systems is presented in Section II. Section III introduces models of DPSs. Section IV presents the observer design problem including: observers in infinite and finite-dimensional spaces and adaptive observers. In Section $\mathrm{V}$ observers for second-order temporal systems are introduced, followed by Section VI, which addresses distributed estimation. Section VII concludes the paper.

\section{LUMPED-PARAMETER OBSERVER DESIGN}

In this section, we briefly recall the observer design problem for lumped-parameter systems. Consider the following linear time-invariant system:

$$
\begin{aligned}
\dot{x}(t) & =A x(t)+B u(t) \\
y(t) & =C x(t)
\end{aligned}
$$

where $x(t) \in \mathbb{R}^{n}, u \in \mathbb{R}^{r}$, and $y(t) \in \mathbb{R}^{q}$ are the input, state, and output vector, respectively, and $A, B, C$ are state, input, and output matrices that have appropriate dimensions. The dot over a variable means the time derivative of the variable. The estimate of the state, $\hat{x}(t)$, is obtained as the output of the following observer [9]:

$$
\dot{\hat{x}}(t)=A \hat{x}(t)+B u(t)+K(y(t)-C \hat{x}(t))
$$

where $K \in \mathbb{R}^{n \times q}$ is the observer gain. The corresponding observer design problem is to find $K$ such that the estimation error $e(t)=\hat{x}(t)-x(t)$ asymptotically converges to zero: $\lim _{t \rightarrow \infty} e(t)=0$. This can be achieved if the real parts of all the eigenvalues of $A-K C$ are negative.

\section{Distributed-PARAMETER Systems}

Distributed-parameter systems are modeled by partial differential equations (PDEs). The evolution of states of a PDE is described in an infinite-dimensional space. DPSs are therefore called infinite-dimensional systems [10]. Take the following normalized $1 \mathrm{D}$ heat equation ${ }^{1}$ as an example:

$$
\frac{\partial x(z, t)}{\partial t}=\frac{\partial^{2} x(z, t)}{\partial z^{2}}+u(z, t), \quad z \in(0,1)
$$

\footnotetext{
${ }^{1}$ The normalized $1 \mathrm{D}$ heat equation describes the temperature distribution over a rod with unity length as a function of time under the effect of a heat source.
} 


$$
\begin{aligned}
\frac{\partial x(0, t)}{\partial z} & =\frac{\partial x(1, t)}{\partial z}=0, & & t \geq 0 \\
x(z, 0) & =x_{0}(z), & & z \in[0,1]
\end{aligned}
$$

where $x$ and $u$ are respectively the temperature and the input heat flow, and $z$ is the spatial variable. The first equation describes the dynamics, while the second and third equation are the boundary condition and the initial condition respectively.

A PDE like (3) when expanded with an output equation can also be expressed as an abstract state space equation:

$$
\begin{aligned}
& \dot{x}(t)=A x(t)+B u(t) \\
& y(t)=C x(t), x(0)=x_{0} .
\end{aligned}
$$

The $A, B, C$ above are defined on a normed linear space [11]. It should be noted that $A, B, C$ are operators and they have different meaning from those in (1). For (3), this can be done by taking the trajectory segment $x(\cdot, t)=\{x(z, t) \mid$ $0 \leq z \leq 1\}$ as the state and by identifying the state space $\mathcal{X}$ with the normed space $L_{2}(0,1)$ of functions $x(\cdot, t)$ that are square-integrable on the spatial interval $[0,1]$ with $\|x(\cdot, t)\|=\left(\int_{0}^{1}|x(z, t)|^{2} \mathrm{~d} z\right)^{\frac{1}{2}}$ [11]. Define the operators $A$ and $B$ on $\mathcal{X}$ to be

$$
\begin{aligned}
A & =\frac{\mathrm{d}^{2}}{\mathrm{~d} z^{2}} \quad \text { with the domain } \\
\mathcal{D}(A)= & \left\{h \in L_{2}(0,1) \mid h, \frac{\mathrm{d} h}{\mathrm{~d} z}\right. \text { are absolutely continuous, } \\
& \left.\frac{\mathrm{d}^{2} h}{\mathrm{~d} z^{2}} \in L_{2}(0,1) \text { and } \frac{\mathrm{d} h(0)}{\mathrm{d} z}=\frac{\mathrm{d} h(1)}{\mathrm{d} z}=0\right\} \\
B=I &
\end{aligned}
$$

where $\mathcal{D}(A)$ is the domain of $A, I$ is the identity, and the function $x_{0}(\cdot, 0) \in L_{2}(0,1)$ is the initial state. The output operator $C$ is determined later since it depends of sensor locations.

The input trajectory $u(\cdot, t)$ and output trajectory $y(\cdot, t)$ are defined similarly to the state. The abstract model simplifies the representation of the PDE model by incorporating the boundary condition into the definition of the domain of $A$, $\mathcal{D}(A)$.

For the purpose of observer and controller design, the dimension of the system must be reduced. This process is called lumping and there are two kinds of lumping [8], [12]: early lumping and late lumping.

In early lumping, the first step of the design process is to reduce the system dimensionality using spatial approximation methods, e.g., finite-difference or finite-element methods. The dimension reduction step results in a finite-dimensional system of ODEs that serves as the basis for observer design. Next, temporal discretization can be applied to obtain discrete-time system models.

In late lumping, the infinite-dimensional model is used during analysis and design. The resulting observer has an infinite number of dimensions, which are then lumped for the implementation.

Several order reduction methods can be used for both early and late lumping. They are Galerkin approximation [13], proper orthogonal decomposition [14], and eigenfunction expansion [15].

\section{Distributed-PARAmeter ObSerVers}

In the literature, there are several approaches to observer design for DPSs. The first approach uses the PDE model (3) and makes use of the available analysis methods for PDEs. The second approach uses the abstract model (4) and applies the existing analysis tools in functional analysis.

For the PDE model (3) the observers have the following form [8]:

$$
\begin{aligned}
\frac{\partial \hat{x}(z, t)}{\partial t} & =\frac{\partial^{2} \hat{x}(z, t)}{\partial z^{2}}+u(z, t) \\
\quad+\ell(z)(\hat{x}(z, t)-x(z, t)), z \in(0,1) & \\
\frac{\partial \hat{x}(z, t)}{\partial z} & =\ell_{\mathrm{b}}(z)(\hat{x}(z, t)-x(z, t)), t \geq 0, z \in\{0,1\} \\
\hat{x}(z, 0) & =\hat{x}_{0}(z), \quad z \in[0,1]
\end{aligned}
$$

where $\ell(z)$ and $\ell_{\mathrm{b}}(z)$ are the observer gains. Inserting (3) and (5) into the error equation:

$$
e(z, t)=\hat{x}(z, t)-x(z, t)
$$

results in

$$
\begin{aligned}
\frac{\partial e(z, t)}{\partial t} & =\frac{\partial^{2} e(z, t)}{\partial z^{2}}+\ell(z) e(z, t), & & z \in(0,1) \\
\frac{\partial \hat{e}(z, t)}{\partial z} & =\ell_{\mathrm{b}}(z) e(z, t), \quad t \geq 0 & & z \in\{0,1\} \\
e(z, 0) & =e_{0}(z)=\hat{x}_{0}(z)-x_{0}(z), & & z \in[0,1]
\end{aligned}
$$

The observer design problem is to determine the observer gains such that the estimation error goes to zero

$$
\lim _{t \rightarrow \infty} e(z, t)=0, \quad z \in[0,1]
$$

For model (4), the observer has the following form

$$
\begin{aligned}
\dot{\hat{x}}(t) & =A \hat{x}(t)+B u(t)+K(y(t)-C \hat{x}(t)) \\
\hat{x}(0) & =\hat{x}_{0}
\end{aligned}
$$

The observer design problem involves finding an observer gain operator $K$ that makes the estimation error $e$, whose evolution is described by

$$
\begin{aligned}
\dot{e}(t) & =(A-K C) e(t) \\
e(0) & =\hat{x}_{0}-x_{0},
\end{aligned}
$$

asymptotically tend to zero.

We can now proceed to an overview of observer design methods for DPSs. A taxonomy of observers for linear DPSs is shown in Figure 1. Note that for infinite-dimensional systems, beside the observer design techniques, the existence of the observer has also been investigated.

\section{A. Finite-Dimensional Observers}

Finite-dimensional observers are observers that are designed using the early-lumping approach. The lumping results in a system of ODEs and makes the observer design methods similar to the case of lumped-parameter systems, but they often include additional elements such as the influence of sensor locations. 


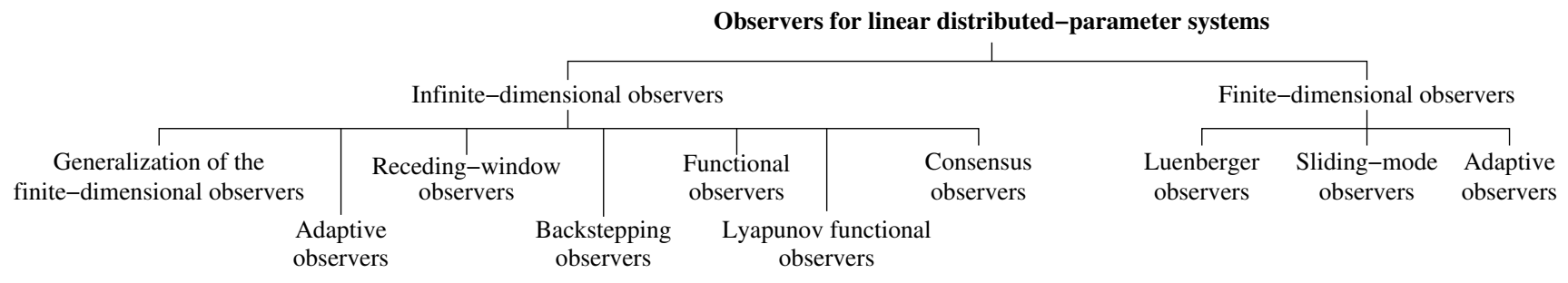

Fig. 1. Overview of observer design approaches for linear DPSs.

Orner and Foster [13] have designed an optimal controller for a system modeled by a PDE. The order of the model is reduced using the Galerkin approximation ${ }^{2}$. The observer is designed to include the influence of the sensor location by optimizing the term $A-K C$ in (2).

Stavroulakis and Sarachik [15] have developed an observer for an optimal control system in which the eigenfunction expansion is used to reduce the order of the model. The observer design follows the method proposed in [16], in which it has been shown that eigenvalues of observers used for optimal control systems cannot be assigned arbitrarily and a systematic design approach has also been given.

An observer that estimates the states of the system and unknown input functions using output measurements has been introduced by Kobayashi and Hitotsuya [17]. In [17], it is assumed that the system in Hilbert space $H$ is decomposable into an $N$-dimensional part:

$$
\dot{x}_{\mathrm{f}}(t)=A_{\mathrm{f}} x_{\mathrm{f}}(t)+P F(t), \quad x_{\mathrm{f}}(0)=x_{\mathrm{f}, 0}
$$

and an infinite-dimensional part:

$$
\dot{x}_{\mathrm{i}}(t)=A_{\mathrm{i}} x_{\mathrm{i}}(t)+Q F(t), \quad x_{\mathrm{i}}(0)=x_{\mathrm{i}, 0}
$$

where $x_{\mathrm{f}}$ and $x_{\mathrm{i}}$ are the state variables of the finite-dimensional part and the infinite-dimensional part, $P$ and $Q$ are orthogonal projections such that $H=P H+Q H$ and $Q=I-P, A_{\mathrm{f}}$ and $A_{\mathrm{i}}$ are the restrictions of $A$ to $P H$ and $Q H$ respectively, and $F(t)$ is the unknown input function. An observer for the $N$-dimensional part has the following form:

$$
\dot{\hat{x}}_{\mathrm{f}}(t)=R \hat{x}_{\mathrm{f}}(t)+S y(t), \quad \hat{x}_{\mathrm{f}}(0)=x_{\mathrm{f}, 0}
$$

for continuous linear operators $R$ and $S$ where $y(t)=$ $M x_{\mathrm{f}}(t)+M x_{\mathrm{i}}(t)$ is the measurement equation and where $R$ fulfills the observer condition [18]: $R=A_{\mathrm{f}}-S M$. Using the solution of the dynamic error equation and assuming that the projected system is observable, the eigenvalues of $R$ can be assigned arbitrarily. The estimated states are then used to approximate the input function $F(t)$. In [19] Kobayashi has investigated the same problem but for a discrete-time system and unknown initial states.

Efe [14] has designed a sliding-mode observer for a reducedorder model obtained by proper orthogonal decomposition. The stability and convergence of the observer are proved using Lyapunov stability conditions.

\footnotetext{
${ }^{2}$ Galerkin methods are a class of methods for converting a continuous operator problem (such as a differential equation) into a discrete problem.
}

\section{B. Infinite-Dimensional Observers}

When the observer design takes the late-lumping approach, the resulting observer has an infinite number of dimensions and it has the form of (5) or (8).

1) Generalization of the observer theory for finitedimensional systems: One of the fundamental problems associated with the abstract model (4) is the generalization of observer theory for finite-dimensional systems to the infinitedimensional case. We can see that the observer equations (8) are the same as those of finite-dimensional systems. However, we cannot directly apply the finite-dimensional observer equations to infinite-dimensional systems because of the different underlying space on which the operators are defined in the infinite-dimensional case [5].

To generalize observer theory to infinite-dimensional systems, Kitamura et al. [20] introduced conditions for the existence and realizability of the observer (8) with no input. The full-order and reduced-order case are also considered with two types of measurements: spatially continuous and spatially discrete.

Gressang and Lamont [21] have investigated another generalization of (4), including a class of linear functional differential equations. In that paper, observability has been proven as a sufficient condition to design full-order and reduced-order observers. It also shown that the eigenvalues of an observerbased state feedback system are the union of the eigenvalues of the closed-loop system and of the observer. However, the generalization does not allow the observer to have arbitrary eigenvalues.

Sakawa and Matsushita [22] have investigated the feedback stabilization of parabolic PDEs and proved that controllability and observability are necessary and sufficient conditions to stabilize the system. This result is applied to the observation problem and it can be shown that observability guarantees the observers to converge.

An extension to the Luenberger observer in relation with sensor locations in DPSs has been studied by El Jai and Amouroux [23]. This study has shown that sensor configurations have a relation with the detectability of the system and the existence of the observer. This means that there are certain sensor configurations that allow the observers to exist and, at the same time, there are also certain sensor configurations for which the observer does not exist.

2) Observer Design: Several observer design methods can be found in the literature. Liu and Lapidus [24] have intro- 
duced a Lyapunov-based observer design for system (4) under the assumption that there are no inputs. The gain operator $K$ can be obtained by first defining a Lyapunov functional based on a norm of the estimation error and next applying the Lyapunov stability condition to this Lyapunov functional.

Yaz et al. [25] have proposed a type of observers called receding-window observers. The observer is in the form (8) with the following observer gain calculation

$$
K\left(P_{N-1}\right)=A P_{N-1} C^{\mathrm{T}}\left(C P_{N-1} C^{\mathrm{T}}+R\right)^{-1}
$$

where $N$ is a positive integer and where $P_{N}$ is computed by iterating the following Riccati and gain equations:

$$
\begin{aligned}
K\left(P_{k}\right)= & A P_{k} C^{\mathrm{T}}\left(C P_{k} C^{\mathrm{T}}+R\right)^{-1} \\
P_{k+1}= & \left(A-K\left(P_{k}\right) C\right) P_{k}\left(A-K\left(P_{k}\right) C\right)^{\mathrm{T}} \\
& +K\left(P_{k}\right) R K\left(P_{k}\right)^{\mathrm{T}}+Q
\end{aligned}
$$

with $k=0, \ldots, N-1, P_{0}, R$, and $Q$ positive definite operators, and $N$ the window length of the observer. In [25] the convergence of the observer and its bounded noise rejection and robustness to parameter perturbation properties are proved. We can see from (10) and (11) that the observer is similar to the Kalman filter (with $R$ and $Q$ the covariance matrices of respectively the measurement noise and the process noise).

Another observer design method has been introduced by Smyshlyaev and Krstic [26] for boundary observation and control $^{3}$ of a class of parabolic PDEs (3). The observer is called backstepping observer because a backstepping-like transformation $^{4}$ is applied to the estimation error $e(z, t)$. It has been shown that the observer gain computation in the new coordinates ensures the stability of the observer in the original coordinates. Miranda et al. [27] have added a sliding-mode term to the observer to obtain a sliding-mode observer.

A prime application of observers in control systems involves feedback stabilization. A functional observer design has been proposed by Fujii [28] for feedback stabilization for boundary control. The resulting observer involves convolution of the input, which results in an infinite-dimensional observer.

The backstepping observer in [26] is also applied to feedback stabilization using boundary control, where the situations of collocated and anti-collocated ${ }^{5}$ actuator and sensor are considered. A similar approach is taken in [29] for the slidingmode control problem in a non-collocated actuator and sensor case.

Köhne [30] designed an observer for a heated-slab set-up by late lumping. The parameters of the observer are calculated via eigenfunction expansion of the dynamic estimation error equation.

\footnotetext{
${ }^{3}$ Boundary control and/or observation systems are a class of DPSs in which the actuation and/or observation is performed at the spatial boundary of the system.

${ }^{4}$ The backstepping-like transformation converts a system into another stable system using an invertible kernel-based transformation.

${ }^{5}$ For a 1D heated bar, "anti-collocated" that means the heater is at one end and the sensor is at the other end.
}

\section{Adaptive Observers}

For systems with unknown parameters, adaptive observers are used to simultaneously reconstruct the states and asymptotically identify the parameters of the system. Results in this topic for lumped-parameter systems can be traced back to the beginning of the 1970s [31].

For the application of adaptive observer for DPSs, in [32], Lilly has extended the result of [33] and shown that a reducedorder model can be used to identify parameters and states of an infinite-dimensional system. It is shown that if the residual energy from the unmodeled dynamics is bounded over a finite interval and the input is persistently exciting, then the estimation will be bounded. This assumes no input boundedness nor plant stability.

Demetriou and Ito [34] have studied adaptive observers of the following form to overcome additive perturbations from output feedback:

$$
\begin{aligned}
& \dot{x}(t)=(A+\Delta) x(t)+B u(t) \\
& y(t)=C x(t)
\end{aligned}
$$

where the matrices $B$ and $C$ have a finite rank, and $\Delta$ is the additive perturbation from output feedback. Several cases of $\Delta$, assuming the same input-output locations (collocation), are presented and for each case an adaptation law obtained using the Lyapunov redesign method [35] is given.

Curtain et al. [36] have extended the results of [34] for the non-collocated case. Reference [37] addresses the case of timevarying input parameters. The adaptation laws are obtained by using the Lyapunov redesign method [35].

Demetriou et al. [38] have proposed an adaptive fault detection observer to monitor and accommodate actuator faults. When the actuator fault occurs, the observer generates a nonzero residual signal. After the fault is detected, the residual signal is also used to automatically reconfigure the system.

\section{SECOnd-Order Distributed-PARAmeter Systems}

Flexible structures and vibration are examples of DPSs that are modeled as second-order time derivative systems [39] that can be written as [40]:

$$
\begin{array}{r}
\ddot{x}(t)+D \dot{x}(t)+F x(t)=B u(t) \\
y(t)=C\left[\begin{array}{ll}
x^{\mathrm{T}}(t) & \dot{x}^{\mathrm{T}}(t)
\end{array}\right]^{\mathrm{T}} \\
x(0)=z_{0} \quad \dot{x}(0)=v_{0}
\end{array}
$$

where $x$ and $\dot{x}$ are the position and velocity states respectively, $D$ is the damping operator, $F$ is the stiffness operator, and $C$ is the output operator.

While (13) can be transformed into a standard state equation $\dot{x}(t)=A x(t)+B u(t)$ and uses a Luenberger observer, to keep the structure, Demetriou [41] has designed the observer directly in the second-order form instead of designing it from the first-order representation. The method also modified the output equation by

$$
y(t)=\left[\begin{array}{ll}
y_{\mathrm{p}}^{\mathrm{T}}(t) & y_{\mathrm{v}}^{\mathrm{T}}(t)
\end{array}\right]^{\mathrm{T}}=\left[\begin{array}{ll}
x^{\mathrm{T}}(t) C_{\mathrm{p}}^{\mathrm{T}} & \dot{x}^{\mathrm{T}}(t) C_{\mathrm{v}}^{\mathrm{T}}
\end{array}\right]^{\mathrm{T}}
$$


where $C_{\mathrm{p}}$ and $C_{\mathrm{v}}$ are matrices with suitable dimensions. Then the observer is designed using the following form

$$
\begin{aligned}
\ddot{\hat{x}}(t)+ & D \dot{\hat{x}}(t)+F \hat{x}(t)=B u(t) \\
& +K_{\mathrm{p}}\left(y_{\mathrm{p}}(t)-C_{\mathrm{p}} \hat{x}(t)\right)+K_{\mathrm{v}}\left(y_{\mathrm{v}}^{\mathrm{T}}(t)-C_{\mathrm{v}} \dot{\hat{x}}(t)\right)
\end{aligned}
$$

where $K_{\mathrm{p}}$ and $K_{\mathrm{v}}$ are the observer gains. This type of observers are called natural observers [41]. In [41], the abstract representation along with the stability and convergence analysis are presented. It can be seen that the observer is basically a Luenberger-type observer with a separate state gain for the velocity and position states. Another second-order observer method that allows observing systems with a positive semidefinite damping was proposed in [42].

The solution to (13a) can be calculated using eigenfunction expansion:

$$
x(t)=\sum_{i=1}^{M} x_{i}(t) \phi_{i}
$$

where $\phi_{i}$ is the eigenvector corresponding to $i$ th eigenvalue that corresponds to $i$ th mode of the system. Theoretically $M=$ $\infty$, but it is usually set to a finite number, say $J$, since in practice actuators and sensors are not able to deal with very high frequencies. The high-frequency modes $\sum_{i=J+1}^{\infty} x_{i}(t) \phi_{i}$ are called the residual or spillover [43].

When the frequencies of the modeled system and its residual are sufficiently separated, the measurement signals are filtered using low-pass filters to remove high-frequency components. However, the separation principle does not apply anymore in this case. Instead of filtering the sensor output, Chait and Radcliffe [44] filtered the estimation error and the filter is included in the observer design, which results in an augmented observer. In [45], an observer has been designed as a part of a vibration control that is robust to the spillover.

\section{Distributed Estimation}

A recent development in the area of state estimation for DPSs is distributed estimation using sensor networks as measurement system. Sensor networks consist of several sensor nodes where each node has embedded computation, communication, and power modules. A node acts as a local observer that computes estimates using its own model and measurements. The communication module allows the sensor nodes to share information with other nodes in the network within a specified communication topology.

Demetriou [46] has proposed a distributed Luenberger observer using sensor network with the consensus method [47]. Each node $i$ in the network has a model similar to (4) but with a modified measurement equation to reflect the sensor node index

$$
y_{i}(t)=C_{i} x_{i}(t)
$$

for $i \in\{1, \ldots, N\}$ where $N$ is the number of nodes in the network. For each node $i$, the following local observer is applied

$$
\dot{\hat{x}}_{i}(t)=A_{i} \hat{x}(t)+B u(t)+G_{i} y_{i}(t)
$$

where $G_{i}$ denotes the observer gain and it is such $A_{i}=A-$ $G_{i} C_{i}$ generates a stable semigroup. The distributed observer with consensus for node $i$ can be written as

$$
\begin{aligned}
\dot{\hat{x}}_{i}(t)=A_{i} \hat{x}(t)+B u(t) & +G_{i} y_{i}(t) \\
& +\alpha_{i} \Phi_{i} \sum_{j=1, i \neq j}^{N}\left(\hat{x}_{j}(t)-\hat{x}_{i}(t)\right)
\end{aligned}
$$

where $\Phi_{i}$ is the consensus operator gain and $\alpha_{i}$ is an additional weighting term. In [46], an adaptive consensus observer has also been introduced in which the consensus term $\alpha_{i} \Phi_{i}$ is made adaptive.

Consensus filters are also proposed in [40] for second-order DPSs based on Luenberger-type observers, resulting in a filter called natural Luenberger consensus filter.

\section{Summary AND CHALlEnges}

We have presented a review of state observer design methods for first-order and second-order linear distributed-parameter systems (DPSs). We have addressed linear observers for infinite-dimensional and finite-dimensional systems. In addition, adaptive methods and distributed state estimation methods for sensor networks have been presented.

The observer design problem in DPSs is more complex than for lumped-parameter systems due to the infinitedimensionality nature of the problem. At the same time, this field is less explored compared to that of lumped-parameter systems. Given the increasing importance of DPSs in current and future applications, we can identify several challenges.

The first is robustness of the observer against model uncertainty. A number of results are available for robust design of lumped-parameter observers, e.g., [48], [49], but so far only a few of them have been extended to the distributed-parameter setting.

Another challenge is discrete-time observer analysis and design. It is true that spatially-temporally discretized DPSs can be considered as (high-dimensional) lumped-parameter systems. However, in this case the spatially neighboring states have strongly influence one another. Beside stability and convergence analysis, for a spatially large system, the spatiallytemporally discretized system will have a large number elements in the state vector. Fast and reliable numerical computation methods are therefore of great importance for this field.

The observer design methods presented in this paper can be applied, for instance, to estimate the temperature and humidity distribution in a greenhouse or state estimation of a flexiblelink robot [50]. Application of the observer design methods to such systems and performance comparisons over real data sets are topics for future work.

\section{ACKNOWLEDGMENT}

The first author gratefully acknowledges the support of the Government of the Republic of Indonesia, Ministry of Communication and Information Technology. This research is partially funded by the European 7th framework STREP project HD-MPC, contract number INFSO-ICT-223854 and the European 7th Framework Network of Excellence HYCON2. 


\section{REFERENCES}

[1] W. Ray, "Some recent applications of distributed parameter systems theory - a survey," Automatica, vol. 14, no. 3, pp. 281-287, 1978.

[2] D. G. Luenberger, "Observing the state of a linear system," IEEE T. on Mil. Electron., vol. 8, no. 2, pp. 74-80, Apr. 1964.

[3] E. A. Misawa and J. K. Hedrick, "Nonlinear observers-a state-of-theart survey," J. Dyn. Syst.-T. ASME, vol. 111, no. 3, pp. 344-352, 1989.

[4] S. K. Spurgeon, "Sliding mode observers: A survey," Int. J. Syst. Sci., vol. 39 , no. 8 , pp. 751-764, 2008 .

[5] M. Athans, "Toward a practical theory for distributed parameter systems," IEEE T. Automat. Contr., vol. 15, no. 2, pp. 245-247, Apr. 1970.

[6] D. L. Russell, "Distributed parameter systems: An overview," in Encyclopedia of Life Support Systems (EOLSS): Control Systems, Robotics And Automation. London: EOLSS Publishers, 2003.

[7] K. Morris, "Control of systems governed by partial differential equations," in The Control Handbook. Boca Raton: CRC Press, 2010.

[8] A. Vande Wouwer and M. Zeitz, "State estimation in distributed parameter systems," in Encyclopedia of Life Support Systems (EOLSS): Control Systems, Robotics and Automation. London: EOLSS Publishers, 2003.

[9] B. Friedland, "Observers," in The Control Handbook. Boca Raton: CRC Press, 1996, pp. 607-618.

[10] M. Tucsnak and G. Weiss, Observation and Control for Operator Semigroups. Basel: Birkhäuser, 2009.

[11] R. Curtain and H. Zwart, An Introduction to Infinite-Dimensional Linear Systems Theory. New York: Springer-Verlag, 1995, no. 21

[12] W. H. Ray, Advanced Process Control. New York: McGraw-Hill, 1981

[13] P. A. Orner and A. M. Foster, "A design procedure for a class of distributed parameter control systems," J. Dyn. Syst.-T. ASME, vol. 93 no. 2, pp. 86-92, 1971.

[14] M. Efe, "A finite dimensional sliding mode observer for a spatially continuous process," in Proc. Int. Workshop Var. Struc. Syst., Antalya Turkey, Jun. 2008, pp. 302-307.

[15] P. Stavroulakis and P. E. Sarachik, "Design of optimal controllers for distributed systems using finite dimensional state observers," in Proc. IEEE Decis. Contr., vol. 12, San Diego, CA, USA, Dec. 1973, pp. 105109.

[16] Y. Yüksel and J. Bongiorno, J., "Observers for linear multivariable systems with applications," IEEE T. Automat. Contr., vol. 16, no. 6, pp. 603-613, Dec. 1971.

[17] T. Kobayashi and S. Hitotsuya, "Observers and parameter determination for distributed parameter systems," Int. J. Contr., vol. 33, no. 1, pp. 31$50,1981$.

[18] D. Luenberger, "An introduction to observers," IEEE T. Automat. Contr., vol. 16, no. 6, pp. 596-602, Dec. 1971.

[19] T. Kobayashi, "Discrete-time observers and parameter determination for distributed parameter systems with discrete-time input-output data," SIAM J. Control Optim., vol. 21, no. 3, pp. 331-351, 1983.

[20] S. Kitamura, S. Sakairi, and M. Nishimura, "Observer for distributedparameter diffusion systems," Electr. Eng. Jpn, vol. 92, no. 6, pp. 142149, 1972.

[21] R. Gressang and G. Lamont, "Observers for systems characterized by semigroups," IEEE T. Automat. Contr., vol. 20, no. 4, pp. 523-528, Aug. 1975.

[22] Y. Sakawa and T. Matsushita, "Feedback stabilization of a class of distributed systems and construction of a state estimator," IEEE T. Automat. Contr., vol. 20, no. 6, pp. 748-753, Dec. 1975

[23] A. El Jai and M. Amouroux, "Sensors and observers in distributed parameter systems," Int. J. Contr., vol. 47, no. 1, pp. 333-347, 1988.

[24] Y. A. Liu and L. Lapidus, "Observer theory for distributed-parameter systems," Int. J. Syst. Sci., vol. 7, no. 7, pp. 731-742, 1976.

[25] I. Yaz, V. Bakke, and E. Yaz, "Receding window observer and dynamic feedback control of discrete infinite dimensional systems," in Proc. 30th IEEE Decis. Contr., vol. 3, Brighton, UK, Dec. 1991, pp. 3031-3032.

[26] A. Smyshlyaev and M. Krstic, "Backstepping observers for a class of parabolic PDEs,” Syst. \& Control Lett., vol. 54, no. 7, pp. 613-625, 2005.

[27] R. Miranda, I. Chairez, and J. Moreno, "Observer design for a class of parabolic PDE via sliding modes and backstepping," in Proc. Int Workshop Var. Struc. Syst., Mexico City, Mexico, Jun. 2010, pp. 215220.

[28] N. Fujii, "Feedback stabilization of distributed parameter systems by a functional observer," SIAM J. Control Optim., vol. 18, no. 2, pp. 108-120, 1980.
[29] M.-B. Cheng, V. Radisavljevic, and W.-C. Su, "Output-feedback boundary control of an uncertain heat equation with noncollocated observation: A sliding-mode approach," in Proc. C. Ind. Elect. Appl., Taichung, China, Jun. 2010, pp. 2187-2192.

[30] M. Köhne, "Implementation of distributed parameter state observers," in Distributed Parameter Systems: Modelling and Identification. Berlin: Springer, 1978, vol. 1, pp. 310-324.

[31] R. Carroll and D. Lindorff, "An adaptive observer for single-input singleoutput linear systems," IEEE T. Automat. Contr., vol. 18, no. 5, pp. 428435, Oct. 1973.

[32] J. H. Lilly, "Finite-dimensional adaptive observers applied to distributed parameter systems," IEEE T. Automat. Contr., vol. 38, no. 3, pp. 469-474, Mar. 1993

[33] G. Kreisselmeier, "The generation of adaptive law structures for globally convergent adaptive observers," IEEE T. Automat. Contr., vol. 24, no. 3, pp. 510-513, Jun. 1979.

[34] M. Demetriou and K. Ito, "Adaptive observers for a class of infinite dimensional systems," in Proc. 13th IFAC World Congress, vol. K, San Francisco, CA, USA, 1996, pp. 409-413.

[35] H. K. Khalil, Nonlinear Systems, 3rd ed. New Jersey: Prentice Hall, 2002.

[36] R. Curtain, M. Demetriou, and K. Ito, "Adaptive observers for structurally perturbed infinite dimensional systems," in Proc. 36th IEEE Decis. Contr., vol. 1, San Diego, CA , USA, Dec. 1997 1997, pp. 509-514.

[37] _ _ "Adaptive observers for slowly time varying infinite dimensional systems," in Proc. 37th IEEE Decis. Contr., vol. 4, Tampa, FL, USA, Dec. 1998 , pp. $4022-4027$.

[38] M. Demetriou, K. Ito, and R. Smith, "Adaptive monitoring and accommodation of nonlinear actuator faults in positive real infinite dimensional systems," IEEE T. Automat. Contr., vol. 52, no. 12, pp. 2332-2338, Dec. 2007.

[39] L. Meirovitch, Dynamics and Control of Structures. New York: John Wiley \& Sons, 1990.

[40] M. A. Demetriou, "Natural consensus filters for second order infinite dimensional systems," Syst. \& Control Lett., vol. 58, no. 12, pp. 826833, 2009.

[41] — , "Natural second-order observers for second-order distributed parameter systems," Syst. \& Control Lett., vol. 51, no. 3-4, pp. 225-234, 2004.

[42] T. D. Nguyen, "Second-order observers for second-order distributed parameter systems in $\mathbb{R}^{2}$," Syst. \& Control Lett., vol. 57, no. 10, pp. 787-795, 2008.

[43] M. Balas, "Feedback control of flexible systems," IEEE T. Automat. Contr., vol. 23, no. 4, pp. 673-679, Aug. 1978.

[44] Y. Chait and C. J. Radcliffe, "Control of distributed parameter systems with spillover using an augmented observer," in P. Amer. Contr. Conf., Minneapolis, MN, USA, Jun. 1987, pp. 1193-1198.

[45] B.-S. Chen, C.-L. Lin, and F.-B. Hsiao, "Robust observer-based control of a vibrating beam," Proc. IME C J. Mech. Eng. Sci., vol. 205, no. 23, pp. 77-89, 1991

[46] M. A. Demetriou, "Design of consensus and adaptive consensus filters for distributed parameter systems," Automatica, vol. 46, no. 2, pp. 300$311,2010$.

[47] R. Olfati-Saber, J. A. Fax, and R. M. Murray, "Consensus and cooperation in networked multi-agent systems," P. IEEE, vol. 95, no. 1, pp. 215-233, Jan. 2007.

[48] G. Ciccarella, M. D. Mora, and A. Germani, "A robust observer for discrete time nonlinear systems," Syst. \& Control Lett., vol. 24, no. 4, pp. 291-300, 1995.

[49] K. C. Veluvolu, Y. C. Soh, and W. Cao, "Robust discrete-time nonlinear sliding mode state estimation of uncertain nonlinear systems," Int. J. Robust \& Nonlin. Contr., vol. 17, pp. 803-828, 2007.

[50] Y. Li and X. Chen, "End-point sensing and state observation of a flexiblelink robot," IEEE/ASME T. Mechatronics, vol. 6, no. 3, pp. 351-356, Sep. 2001. 